\title{
Mixing quantum and classical mechanics and uniqueness of Planck's constant
}

\author{
Debendranath Sahoo \\ Institute of Physics, P.O. Sainik School, \\ Bhubaneswar 751 005, Orissa, Indid*
}

\begin{abstract}
Observables of quantum or classical mechanics form algebras called quantum or classical Hamilton algebras respectively (Grgin E and Petersen A (1974) J Math Phys 15764 1], Sahoo D (1977) Pramana 8545 2]). We show that the tensor-product of two quantum Hamilton algebras, each characterized by a different Planck's constant is an algebra of the same type characterized by yet another Planck's constant. The algebraic structure of mixed quantum and classical systems is then analyzed by taking the limit of vanishing Planck's constant in one of the component algebras. This approach provides new insight into failures of various formalisms dealing with mixed quantumclassical systems. It shows that in the interacting mixed quantum-classical description, there can be no back-reaction of the quantum system on the classical. A natural algebraic requirement involving restriction of the tensor product of two quantum Hamilton algebras to their components proves that Planck's constant is unique.
\end{abstract}

PACS numbers: 03.65.Fd;03.65.Ta

*dsahoo@iopb.res.in Permanent address: Materials Science Division, Indira Gandhi Centre for Atomic Research, Kalpakkam, Tamil Nadu, PIN: 603 102, India. 


\section{INTRODUCTION}

The title of the present paper may create an impression that two disjoint subjects are being discussed together. However, a little reflection would convince the reader that there is a connecting thread between the two. Inherent in a quantum system is a Planck's constant (PC) governing its behaviour whereas a classical system can be thought of as a system with zero PC. Thus in mixed quantum-classical mechanics, we are dealing with two systems with different PC's. Now in proving the uniqueness of PC, it is but natural to consider two systems with different values of $\mathrm{PC}$ and then to examine the consequences. The fact that two different PC's come into play in the analysis of both the subjects, provides the connecting thread. The purpose of this work is two-fold: to investigate why one can not have a fundamentally satisfactory dynamical description of interacting quantum-classical systems and to understand the uniqueness of $P C$ conventionally assumed in the physics literature. The method of our investigation is of algebraic nature.

As of now there is no consistent theory of interaction of a classical system with a quantum one. Such a theory is desirable since a variety of problems in a number of different fields involve coupling of quantum and classical degrees of freedom. In the development of quantum mechanics (QM), Niels Bohr [3] had always insisted that measuring instruments must be describable in classical terms, but did not provide a theoretical framework for the description of interacting quantum-classical systems. The so-called Copenhagen interpretation of quantum theory, founded on this assumption, is by and large accepted by all physicists; yet this lacuna has remained as a sore point. The issue of a mixed quantum-classical description is important in the discussion of early universe physics where fully quantum matter fields have to be necessarily coupled to the gravitational field which is classical. Traditional approach to this problem has been to couple the gravity field to the expectation values of the quantum energy-momentum tensor of the matter fields. In this approach one misses the effects of quantum fluctuations on the classical gravitational field-the so-called quantum back-reaction.

There has been no dearth of effort in constructing a mathematically consistent theory of

such mixed systems. Some authors [5, 6, 7, 8] use mixed classical-quantum notation to denote the dynamical variables (DV's) of the (mixed) system. Let $x, y, x_{i}, \cdots$ denote classical DV's which are ordinary functions of commuting phase-space variables and let $X, Y, X_{i}, \cdots$ denote 
quantum DV's which are noncommuting operators (acting on some suitable Hilbert space of states. Then typical DV's of the mixed quantum-classical system are denoted as $X x, Y y, \cdots$, which are operator-valued functions. Also the general DV's of the mixed system may be denoted $\mathcal{X}, \mathcal{Y}, \cdots$ where $\mathcal{X}=\sum_{i} X_{i} x_{i}, \mathcal{Y}=\sum_{i} Y_{i} y_{i}$, etc. Let us use the notation

$$
\begin{aligned}
& {[X, Y]^{-}=\frac{(X Y-Y X)}{i \hbar},} \\
& {[X, Y]^{+}=\frac{1}{2}(X Y+Y X),}
\end{aligned}
$$

to denote the commutator and the anticommutator brackets respectively and let $\{x, y\}_{P}$ denote the usual Poisson bracket of classical DV's. In order to denote the corresponding bracket of mixed DV's, let us adopt the notation $[\{\}$,$] . Guided mainly by guess-work,$ Following definitions of this bracket have been proposed:

$$
\begin{aligned}
& {[\{x X, y Y\}]=x y[X, Y]^{-}+\{x, y\}_{P}[X, Y]^{+}} \\
& \text {(Boucher and Traschen }[6]) \\
& {[\{\mathcal{X}, \mathcal{Y}\}]=[\mathcal{X}, \mathcal{Y}]+\frac{1}{2}\left(\{\mathcal{X}, \mathcal{Y}\}_{P}-\{\mathcal{Y}, \mathcal{X}\}_{P}\right)} \\
& (\text { Aleksandrov }[\underline{9}], \text { Caro and Salcedo }[\underline{8}]) \\
& {[\{\mathcal{X}, \mathcal{Y}\}]=[\mathcal{X}, \mathcal{Y}]+\{\mathcal{X}, \mathcal{Y}\}_{P} \quad(\text { Anderson }[\underline{5}])}
\end{aligned}
$$

Caro and Salcedo[8] consider a quantum system consisting of two mutually interacting subsystems and enquire whether it is possible to take the classical limit $(i e$, letting $\hbar \rightarrow 0)$ in just one of the subsystems maintaining at the same time an internally consistent dynamics for the resulting mixed quantum-classical system. They call this as the semi-quantization problem and arrive at the bracket (41). Further, they show that this bracket, although antisymmetric, does not satisfy the Jacobi identity. In his investigation of quantum back-reaction on classical variables, Anderson [5] suggests the bracket (5), which is not even antisymmetric. Prezhdo and Kisil[10] develop a mathematically sophisticated formalism and arrive at the result identical to (3) but written in terms of the symbols of the operators (see their Eq.(24)).

A satisfactory bracket $($, ) describing any dynamics, be it classical or quantum (and also 
desirable for a mixed classical-quantum system), must possess the following properties[11]:

$$
\begin{aligned}
& (A, B)=-(B, A) \quad \text { (antisymmetry) } \\
& ((A, B), C)+((B, C), A)+((C, A), B)=0 \quad \text { (Jacobi identity) } \\
& (A, B C)=(A, B) C+B(A, C) \quad \text { (Derivation identity) }
\end{aligned}
$$

As is well known[8], antisymmetry of the bracket ensures conservation of energy, the Jacobi identity ensures that $(A, B)$ also evolves dynamically and the derivation identity ensures that the product $A B$ also evolves with consistent dynamics. Lack of any of these properties impose severe impediments to mixing of classical and quantum degrees of freedom. The bracket (5) has none of these properties whereas the bracket (3) satisfies neither the Jacobi nor the derivation identity [8, 12]. Diosi and his group[13] extensively investigate the question of coupling of quantum-classical systems focussing their attention mainly on maintaining the positivity of the quantum states. Hay and Peres [14] treat the apparatus quantum mechanically while it interacts with the system and then give a classical description of the apparatus within the framework of the Wigner functions. Peres and Temo[15] develop a hybrid formalism by taking recourse to the Koopman operator representation of classical Hamiltonians and conclude that the correspondence principle is violated due to the interaction. Belavkin and his collaborators [16] develop a stochastic Hamiltonian theory for coupling of a quantum system with an apparatus. In this approach, attention is focussed on providing purely dynamical arguments to derive entanglement, decoherence and collapse of the coupled system consisting of a quantum system and a (semi-classical) apparatus. However, in this theory, as in the original von Neumann theory 17] of measurement, both the system and the apparatus are treated quantum mechanically and a "reduction model" is proposed achieving some improvement over the von Neumann reduction postulate. Some more discussion of this model will be given in the last section dealing with discussion of our results. Sudarshan and his collaborators [18] propose a novel procedure of coupling a classical system (the apparatus) with a quantum one. They embed the classical system into what they term as a classical enlarged quantum system (CEQS). The set of observable phase-space variables (regarded as commuting operators) are supplemented by an equal number of unobservable conjugate variables (noncommuting with the previous set). In this approach, after coupling the CEQS with the quantum system and subsequently decoupling these systems, the value of the measured quantity of the quantum system is transfered to an appropriate observable 
of the CEQS. Certain principle of integrity invoked by these authors, assures satisfactory behaviour of observables of the classical system in the measurement process. However, the state evolution of the CEQS indicates that the classical system does not remain purely classical after the interaction is over. The basic mathematical problem of a truly satisfactory quantum-classical coupling remains unsolved. The question whether there exists a satisfactory bracket for the mixed quantum-classical systems calls for a detailed investigation from a purely algebraic point of view. The present work has this as one of its main motivation.

The second important result which is derived in this work is an algebraic proof that $\mathrm{PC}$ is unique. The possibility of multiplicity of PC's cannot be logically ruled out and its universality is of empirically established nature [19]. Fischback et al [20] have examined this question carefully and after recalling how the existence of several PC's leads to violation of space-time symmetry laws, suggest a possible test for experimentally verifying this assumption. Battaglia 21], while arguing that the introduction of several PC's is undesirable, suggest remedies to some of the embarrassing problems arising in the event that experiments do allow for such an eventuality. Our proof of uniqueness of PC resolves this issue. We now describe the abstract algebraic structures of quantum and classical mechanics.

\section{HAMILTON ALGEBRA}

In order to motivate the definition of the algebra of observables of QM we note that in the von Neumann formulation[17] of QM, the associative algebra $\mathcal{B}(\mathcal{S})$ of bounded linear operators, defined over the complex field $\mathbb{C}$, acts on a Hilbert space $\mathcal{S}$ of states. The set of observables $\overline{\mathcal{B}}(\mathcal{S})$, consisting of self-adjoint elements of $\mathcal{B}(\mathcal{S})$ and defined over the real field $\mathbb{R}$ inherits from $\mathcal{B}(\mathcal{S})$ the structure of a Jordan-Lie algebra 22] with a Jordan product $[X, Y]^{+}$ and a Lie product $[X, Y]^{-}$where $X, Y \in \overline{\mathcal{B}}(\mathcal{S})$. Since every $X \in \mathcal{B}(\mathcal{S})$ can be written uniquely in the form $X=X_{1}+i X_{2}$ with $X_{1}, X_{2} \in \overline{\mathcal{B}}(S)$ and $i=\sqrt{-1}, \mathcal{B}(\mathcal{S})$ is the complex extension of $\overline{\mathcal{B}}(\mathcal{S})$. This observation suggests the definition of a quantum Hamilton algebra (QHA) 2, 4]: it is a two-product algebra $\left\{\mathcal{H}, \alpha^{a}, \sigma^{a}, \mathbb{R}\right\}$, over the real field $\mathbb{R}$, parametrized by a real number $a$ called the quantum constant. Here $\mathcal{H}$ is the linear space underlying the algebra; $\alpha^{a}$ and $\sigma^{a}$ are bilinear products $\alpha^{a}, \sigma^{a}: \mathcal{H} \otimes \mathcal{H} \rightarrow \mathcal{H}$. Henceforth we shall denote this algebra by the notation $\mathcal{H}^{a}$. The correspondence between the symbols and their

abstract counterparts is: $\overline{\mathcal{B}}(\mathcal{S}) \rightarrow \mathcal{H},[,]^{-} \rightarrow \alpha^{a}$, [, $]^{+} \rightarrow \sigma^{a}$ and $a \rightarrow \hbar^{2} / 4$. Elements of the 
set $\mathcal{H}$ are denoted by $e, f, g, h, \cdots$, where $e$ is the unit element of $\mathcal{H}^{a}$ (with respect to $\sigma^{a}$ ). Note that in the algorithmic form, we have earlier used the notation $X, Y$, etc. to denote the (operator) elements of the elements of $\overline{\mathcal{B}}(\mathcal{S})$. A QHA is defined 2, 4] by the identities:

$$
\begin{aligned}
& f \alpha^{a} g=-g \alpha^{a} f \quad(\text { antisymmetry }), \\
& f \alpha^{a}\left(g \alpha^{a} h\right)+g \alpha^{a}\left(h \alpha^{a} f\right)+h \alpha^{a}\left(f \alpha^{a} g\right)=0 \quad(\text { Jacobi identity }), \\
& f \sigma^{a} g=g \sigma^{a} f \quad(\text { symmetry }), \\
& f \alpha^{a}\left(g \sigma^{a} h\right)=\left(f \alpha^{a} g\right) \sigma^{a} h+g \sigma^{a}\left(f \alpha^{a} h\right) \quad\left(\text { derivation of } \alpha^{a} \text { wrt } \sigma^{a}\right), \\
& \Delta_{\sigma}^{a}(f, g, h) \equiv\left(f \sigma^{a} g\right) \sigma^{a} h-f \sigma^{a}\left(g \sigma^{a} h\right) \\
& =a\left[\left(f \alpha^{a} h\right) \alpha^{a} g\right] \quad(\text { Canonical relation, CR }) .
\end{aligned}
$$

The CR can be trivially checked to hold in its algorithmic form in $\overline{\mathcal{B}}(\mathcal{S})$. Elevation of this trivial looking relation in this form to the status of a defining identity of our algebra follows from the composition properties of the HA's which hold if and only if the CR is assumed to hold. Note that both $\sigma^{a}$ and $\alpha^{a}$ are nonassociative products; the nonassociativity of $\sigma^{a}$ is measured by the associator $\Delta_{\sigma^{a}}$, a trilinear object; and the rhs of Eq.(13) can also be written (but for the constant factor $a$ ) as an $\alpha^{a}$-associator. Thus the $\mathrm{CR}$ is an exact relation between the two associators. Note also that the standard Jordan identity [24] $f^{2} \sigma^{a}\left(g \sigma^{a} f\right)=\left(f^{2} \sigma^{a} g\right) \sigma^{a} f$ with $f^{2}=f \sigma^{a} f$ follows from (13) by substituting in it $f=h$ and using (9). It is the interaction of the Lie and the Jordan structures via Eqs. (12) and (13) that makes a QHA an interesting algebraic object in its own right.

A classical Hamilton algebra $(\mathrm{CHA}) \mathcal{H}^{0}=\left\{\mathcal{H}, \alpha^{0}, \sigma^{0}, \mathbb{R}\right\}$ is now defined by setting $a=$ 0 in the identities (92 13). Note that the product $\sigma^{0}$ is associative in addition to being commutative-a property of classical phase space functions. We next turn our attention on the most important characteristic of Hamilton algebras (HA's).

\section{COMPOSITION PROPERTIES OF HAMILTON ALGEBRAS}

Interaction of two quantum systems should result in a composite system describable within the same framework. This intuitive idea is made rigorous by postulating that the tensorproduct (TP) composition of two QHA's is yet another QHA. It can be easily verified that the auxiliary product

$$
\tau^{a}=\sigma^{a}+\sqrt{-a} \alpha^{a}
$$


defined in the complex extension $\mathcal{A}^{a}$ of $\mathcal{H}^{a}, \tau^{a}: \mathcal{A}^{a} \otimes \mathcal{A}^{a} \rightarrow \mathcal{A}^{a}$ is an associative product. Here the detailed form of the above symbolic relation is

$$
f \tau^{a} g=f \sigma^{a} g+\sqrt{-a} f \alpha^{a} g, \quad \text { for } \quad f, g \in \mathcal{A}^{a} .
$$

Note that $\sigma^{a}$ and $\alpha^{a}$ are derived products:

$$
\begin{array}{r}
f \sigma^{a} g=\frac{1}{2}\left(f \tau^{a} g+g \tau^{a} f\right), \\
f \alpha^{a} g=\frac{1}{2 \sqrt{-a}}\left(f \tau^{a} g-g \tau^{a} f\right) .
\end{array}
$$

The algebra $\left\{\mathcal{A}, \tau^{a}, \mathbb{C}\right\}^{a}=\mathcal{A}^{a}$, where $\mathbb{C}$ is the complex field, is the associative envelope algebra of $\mathcal{H}^{a}$. We now follow the standard procedure 23] of forming the tensor product $\mathcal{A}^{a_{12}}$ of $\mathcal{A}^{a_{1}}$ and $\mathcal{A}^{a_{2}}$. The precise definition of $\tau_{12}$ makes use of the "switching map"

$$
\begin{aligned}
& S:\left(\mathcal{A}_{1} \otimes \mathcal{A}_{2}\right) \otimes\left(\mathcal{A}_{1} \otimes \mathcal{A}_{2}\right) \longrightarrow\left(\mathcal{A}_{1} \otimes \mathcal{A}_{1}\right) \otimes\left(\mathcal{A}_{2} \otimes \mathcal{A}_{2}\right), \\
& S:\left(f_{1} \otimes f_{2}\right) \otimes\left(g_{1} \otimes g_{2}\right) \mapsto\left(f_{1} \otimes g_{1}\right) \otimes\left(f_{2} \otimes g_{2}\right) .
\end{aligned}
$$

Then one has

$$
\tau_{12}=\left(\tau_{1} \otimes \tau_{2}\right) \circ S
$$

Here for brevity we have suppressed the superscripts in the $\tau$ 's. Thus $\tau_{1}$ stands for $\tau_{1}^{a_{1}}$, etc. Similar notation will be used for the other products too. The symbol o denotes the composition of maps. For brevity we shall use the notation $f_{12} \equiv f_{1} \otimes f_{2},(f \sigma g)_{12} \equiv$ $f_{12} \sigma_{12} g_{12},\left[\Delta_{\sigma}(f, g, h)\right]_{12} \equiv(f \sigma g)_{12} \sigma_{12} h_{12}-f_{12} \sigma_{12}(g \sigma h)_{12}$, etc. Consider two QHA's $\mathcal{H}_{1}{ }^{a_{1}}$ and $\mathcal{H}_{2}{ }^{a_{2}}$. Let

$$
\begin{aligned}
& \tau_{12}=\sigma_{12}+\sqrt{-a_{12}} \alpha_{12}, \\
& \tau_{k}=\sigma_{k}+\sqrt{-a_{k}} \alpha_{k} \quad(\mathrm{k}=1,2) .
\end{aligned}
$$

Then Eq.(19) implies

$$
\begin{aligned}
& \sigma_{12}+\sqrt{-a_{12}} \alpha_{12}=\left(\sigma_{1}+\sqrt{-a_{1}} \alpha_{1}\right) \otimes\left(\sigma_{2}+\sqrt{-a_{2}} \alpha_{2}\right) \circ S \\
& =\left[\left(\sigma_{1} \otimes \sigma_{2}-\sqrt{a_{1} a_{2}} \alpha_{1} \otimes \alpha_{2}\right)+\left(\sqrt{-a_{1}} \alpha_{1} \otimes \sigma_{2}+\sqrt{-a_{2}} \sigma_{1} \otimes \alpha_{2}\right] .\right.
\end{aligned}
$$

Now equating the symmetric and antisymmetric parts of both sides we obtain the composition properties of the derived products:

$$
\begin{aligned}
\sigma_{12} & =\left[\left(\sigma_{1} \otimes \sigma_{2}\right)-\sqrt{a_{1} a_{2}}\left(\alpha_{1} \otimes \alpha_{2}\right)\right] \circ S, \\
\alpha_{12} & =\left[\left(\sqrt{\frac{a_{1}}{a_{12}}}\left(\alpha_{1} \otimes \sigma_{2}\right)+\sqrt{\frac{a_{2}}{a_{12}}}\left(\sigma_{1} \otimes \alpha_{2}\right)\right)\right] \circ S .
\end{aligned}
$$


Expanded forms of Eqs. (23) and (24) are

$$
\begin{gathered}
(f \sigma g)_{12}=(f \sigma g)_{1} \otimes(f \sigma g)_{2}-\sqrt{a_{1} a_{2}}(f \alpha g)_{1} \otimes(f \alpha g)_{2}, \\
(f \alpha g)_{12}=\left[\left(\sqrt{\frac{a_{1}}{a_{12}}}(f \alpha g)_{1} \otimes(f \sigma g)_{2}+\sqrt{\frac{a_{2}}{a_{12}}}(f \sigma g)_{1} \otimes(f \alpha g)_{2}\right)\right] .
\end{gathered}
$$

With these composition laws of the algebraic products, we now proceed to prove that the identities (9]13) also hold in the algebra $\mathcal{H}^{a_{12}}$. This is a highly nontrivial result since the TP of two algebras in general leads to an algebra not necessarily of the same type. For example, the TP of two Lie algebras has a product which is symmetric contrary to the antisymmetric nature of the Lie product. Let $f_{12}=f_{1} \otimes f_{2}, g_{12}=g_{1} \otimes g_{2}$ and $h_{12}=h_{1} \otimes h_{2}$ denote three arbitrary elements in $\mathcal{H}_{1} \otimes \mathcal{H}_{2}$. For brevity we shall write $f_{1} \otimes f_{2}=f_{1} f_{2}$, etc. We now demonstrate the following results.

Lemma $1 \alpha_{12}$ is antisymmetric.

Proof:

$$
\begin{aligned}
(f \alpha g)_{12} & =\sqrt{\frac{a_{1}}{a_{12}}}(f \alpha g)_{1}(f \sigma g)_{2}+\sqrt{\frac{a_{2}}{a_{12}}}(f \sigma g)_{1}(f \alpha g)_{2} \\
& =-\sqrt{\frac{a_{1}}{a_{12}}}(g \alpha f)_{1}(g \sigma f)_{2}-\sqrt{\frac{a_{2}}{a_{12}}}(g \sigma f)_{1}(g \alpha f)_{2} \\
& =-(g \alpha f)_{12} .
\end{aligned}
$$

Here in the second line, antisymmetry of $\alpha_{k}(k=1,2)(\mathrm{Eq} 9)$ and the commutativity of $\sigma_{k}$ (Eq11) have been used.

Proceeding similarly we have

Lemma $2 \sigma_{12}$ is symmetric.

Lemma $3 \alpha_{12}$ satisfies the Jacobi identity 
Proof:

$$
\begin{aligned}
((f \alpha g) \alpha h))_{12} & =\left[\sqrt{\frac{a_{1}}{a_{12}}}(f \alpha g)_{1}(f \sigma g)_{2}+\sqrt{\frac{a_{2}}{a_{12}}}(f \sigma g)_{1}(f \alpha g)_{2}\right] \alpha h_{12} \\
& =\sqrt{\frac{a_{1}}{a_{12}}}\left[\sqrt{\frac{a_{1}}{a_{12}}}((f \alpha g) \alpha h)_{1}((f \sigma g) \sigma h)_{2}+\sqrt{\frac{a_{2}}{a_{12}}}((f \alpha g) \sigma h)_{1}((f \sigma g) \alpha h)_{2}\right] \\
& +\sqrt{\frac{a_{2}}{a_{12}}}\left[\sqrt{\frac{a_{1}}{a_{12}}}((f \sigma g) \alpha h)_{1}((f \alpha g) \sigma h)_{2}+\sqrt{\frac{a_{2}}{a_{12}}}((f \sigma g) \sigma h)_{1}((f \alpha g) \alpha h)_{2}\right] \\
& =-\frac{1}{a_{12}}\left[\left(\Delta_{\sigma}(g, h, f)\right)_{1}((f \sigma g) \sigma h)_{2}+((f \sigma g) \sigma h)_{1}\left(\Delta_{\sigma}(g, h, f)\right)_{2}\right] \\
& \left.+\frac{\sqrt{a_{1} a_{2}}}{a_{12}}((f \alpha g) \sigma h)_{1}\left\{\sqrt{\frac{a_{1}}{a_{12}}}(f \alpha h) \sigma g\right)_{2}+\sqrt{\frac{a_{2}}{a_{12}}}(f \sigma(g \alpha h))_{2}\right\} \\
& \left.+\frac{\sqrt{a_{1} a_{2}}}{a_{12}}\left\{\sqrt{\frac{a_{1}}{a_{12}}}(f \alpha h) \sigma g\right)_{1}+\sqrt{\frac{a_{2}}{a_{12}}}(f \sigma(g \alpha h))_{1}\right\}((f \alpha g) \sigma h)_{2} .
\end{aligned}
$$

Here in the third equality, the CR (13) has been used in the first two terms and the derivation property (12), in the last two terms. Cyclically permuting $f, g, h$ in this relation leads to two similar relations summing which results in separate cancellation of all terms with the

prefactor $-\frac{1}{a_{12}}$ and those with the prefactor $\frac{\sqrt{a_{1} a_{2}}}{a_{12}}$, thus proving the Jacobi identity.

We state two other lemmas the proofs of which are relegated to the appendix because they are lengthy.

Lemma $4 \alpha_{12}$ is a derivation wrt $\sigma_{12}$, i e the identity (12) is satisfied in the TP space.

Lemma $5 \quad \sigma_{12}$ and $\alpha_{12}$ are related by the $C R$ (13).

Now in view of the lemmas 1-5, we have

Theorem 1 The algebra $\mathcal{H}^{a_{12}}$ is a $Q H A$.

This is our main result. This result can be interpreted in the following way. Suppose one starts with two physical systems each describable by its Hamiltonian and its own collection of observables satisfying the evolution given by its Lie bracket $\alpha$ and satisfying the properties expressed by the identities (9, 13). The two systems may require different PC's for their complete (algebraic) description. Yet their composite is describable by an 'interaction' Hamiltonian along with its other observables following the evolution by a Lie bracket and also satisfying the same identities (9, 13) and with a PC which is in principle different from the PC's associated with the components. Thus two quantum systems with different PC's can in principle interact in a scheme which provides for a consistent dynamics. We shall 
however show that another natural algebraic requirement restricts this possibility further. Implications of this theorem is described in the next section.

Before ending this section, we note the composition laws of $\sigma$ and $\alpha$ products for the special case for which the component HA's and their TP are all characterized by the same quantum constant $a$ :

$$
\begin{aligned}
& (f \sigma g)_{12}=(f \sigma g)_{1} \otimes(f \sigma g)_{2}-a(f \alpha g)_{1} \otimes(f \alpha g)_{2} \\
& (f \alpha g)_{12}=(f \alpha g)_{1} \otimes(f \sigma g)_{2}+(f \sigma g)_{1} \otimes(f \alpha g)_{2} .
\end{aligned}
$$

These laws were earlier derived in refs. 11, 4]. We also note two interesting facts: (a) whereas the composition law of $\sigma$ alone depends on $a$, that of $\alpha$ is independent of $a$ and (b) The composition law for the $\alpha^{0}$ product in a CHA is identical to that of the $\alpha^{a}$ product in a QHA. We now turn to the treatment of mixed quantum and classical HA's.

\section{MIXED QUANTUM-CLASSICAL HAMILTON ALGEBRA}

A hybrid quantum-classical system is of considerable interest from the point of view of quantum measurement theory. In the orthodox Copenhagen philosophy of measurement, in order to measure an observable pertaining to a quantum system, one has to couple a classical system (the apparatus) with it for a certain duration of time during which the measurement takes place and subsequently the latter is decoupled from the former. A measurement is achieved if unambiguous information concerning the value of the measured variable is transfered (or "stored") into some suitable observable of the apparatus and thus one obtains this information (the "pointer reading") after the decoupling is over. This transfer process is technically referred to as the back-reaction of the quantum system. Theoretically one achieves it by using a coupling Hamiltonian in the TP space of observables of both the systems. It is but natural to look for a Lie product in the TP space which must be constructed out of the Lie products of the component algebras, one of which is the commutator bracket of operators (for the quantum system) and the other, the Poisson bracket of the phase-space functions (for the classical system). Rather than banking upon the correct relation (26), one is tempted to be guided by the inappropriate relation (30). In this relation, if one regards the component 1 as being quantum and 2 being classical, one would be naturally led to the mixed bracket (3) with appropriate algorithmic identifications. Note that from our point 
of view, this would be an illegal procedure not permitted in our analysis. Misuse of (30) is the reason why the bracket (3) and equivalently, the bracket (41) does not satisfy the Jacobi identity-a fact which has been explicitly checked in reference[8]. It is the presence of the second term in the rhs of Eq.(30) which affects change in the classical variable. This is precisely the term responsible for the back-reaction of the system 1 (quantum) on the system 2 (classical).

A simple example to illustrate the back-reaction concept can be given for more clarification. Consider two quantum free particle systems labeled $k=1,2$ with masses $m_{k}$ and momenta operators $\hat{p}_{k}$. Their Hamiltonians are given by $\hat{h}_{k}=\hat{p}_{k}^{2} / 2 m_{k}$. Let us be interested in measuring $\hat{p}_{1}$. A convenient coupling Hamiltonian for this purpose is $\hat{h}_{12}=g(t) \hat{p}_{1} \otimes \hat{x}_{2}$ where $\hat{x}_{2}$ is the position variable of the second particle and $g(t)$ is a coupling parameter such that it is everywhere zero, except between $t_{0}$ and $t_{0}+\Delta t$ (the duration of measurement), where it is constant $\left(=g_{0}\right)$. Confirming to our notation, we have the full Hamiltonian

$$
\hat{h}_{12}=\frac{\hat{p}_{1}^{2}}{2 m_{1}} \otimes \hat{I}_{2}+\hat{I}_{1} \otimes \frac{\hat{p}_{2}^{2}}{2 m_{2}}+g(t) \hat{p}_{1} \otimes \hat{x}_{2} .
$$

Here $\hat{I}_{k}$ is the unit element of the QHA $\mathcal{H}^{k}$. Following are the equations of motion, dictated by Eq.(30):

$$
\begin{aligned}
& \dot{\hat{p}}_{1}=0, \\
& \dot{\hat{x}}_{1}=\frac{\hat{p}_{1}}{m_{2}}+g(t) \hat{x}_{2}, \\
& \dot{\hat{p}}_{2}=-g(t) \hat{p}_{1}, \\
& \dot{\hat{x}}_{2}=\frac{\hat{p}_{2}}{m_{2}}
\end{aligned}
$$

Here $t$ is the time parameter and the time derivative is denoted by an overdot. We treat the system 1 as one whose momentum $\hat{p}_{1}$ is to be measured and the system 2 as the (quantum) apparatus. The first equation implies that $\hat{p}_{1}$ does not change as a result of the measurement. On solving for $\hat{p}_{2}$, we obtain

$$
\hat{p}_{2}-\hat{p}_{2}^{0}=-g_{0} \hat{p}_{1} \Delta t
$$

This equation implies a correlation between $\hat{p}_{2}-\hat{p}_{2}^{0}$ and $\hat{p}_{1}$, such that if $\hat{p}_{2}-\hat{p}_{2}^{0}$ is observed, one can calculate $\hat{p}_{1}$. This change in momentum of the second system arises due to the back-reaction of the first system. This illustrates how the information transfer between the system and the apparatus takes place in the von Neumann theory 17] of measurement. 
We now consider strictly the Bohr point of view that the apparatus is got to be a classical system. Suppose that in the above example the system 1 is quantum, but the system 2 is classical. then it is no longer correct to use Eq.(30). Instead, one should use relation (26). Let $a_{1}=\hbar_{1}^{2} / 4, a_{2}=0$ and let $a_{12}=\hbar_{12}^{2} / 4$. We then obtain immediately from Eqs.(2324) the results for the mixed products:

$$
\begin{aligned}
\sigma_{12} & =\left(\sigma_{1} \otimes \sigma_{2}\right) \circ S, \\
\alpha_{12} & =\frac{\hbar}{\hbar_{12}}\left(\alpha_{1} \otimes \sigma_{2}\right) \circ S .
\end{aligned}
$$

The constant $\hbar_{12}$ being arbitrary, we have to decide on its value from some other consideration. The value $\hbar_{12}=0$ does not lead to any meaningful structure whereas the choice $\hbar_{12}=\hbar$ would mean that $\mathcal{H}^{a} \otimes \mathcal{H}^{0}\left(a=\hbar^{2} / 4\right)$ is a QHA. It would mean that a mixed quantumclassical system is a quantum system governed by a Lie bracket $\alpha_{12}=\left(\alpha_{1}^{a} \otimes \sigma_{2}^{0}\right) \circ S$. We therefore have

$$
\begin{aligned}
& \left(f_{1} \otimes f_{2}\right) \alpha_{12}\left(g_{1} \otimes g_{2}\right)=\left(f \alpha^{a} g\right)_{1} \otimes\left(f \sigma^{0} g\right)_{2} \\
& \left(f_{1} \otimes f_{2}\right) \sigma_{12}\left(g_{1} \otimes g_{2}\right)=\left(f \sigma^{a} g\right)_{1} \otimes\left(f \sigma^{0} g\right)_{2}
\end{aligned}
$$

In algorithmic form, these brackets are

$$
\begin{aligned}
& {[\{X x, Y y\}]^{-}=[X, Y]^{-} x y,} \\
& {[\{X x, Y y\}]^{+}=[X, Y]^{+} x y .}
\end{aligned}
$$

Comparison Eqs. (36) and (30) reveals that the second term in the rhs of (30) is no longer present in the rhs of (36). Following through the example of measurement given above it is clear that the absence of the product $\alpha_{2}$ in the composition law (36) leads to "freezing" of classical dynamics. In other words there is no back-reaction effect of the quantum system on the classical. This is a no go result. Clearly, it does not mean that interactions between classical and quantum systems vanish. Interactions exist and are given by elements belonging to the tensor product algebra $\mathcal{H}^{a} \otimes \mathcal{H}^{0}$. These, however, do not affect any change in the classical "pointer" variable! This pinpoints the root cause of the impediment to a satisfactory description of dynamical evolution of a mixed quantum-classical system. No wonder that the standard Lie bracket [5, 6, 10] for mixed quantum-classical system, suggested along the line of Eq.(30), is not compatible with the algebraic requirement. This explains the futility of such approaches. We now turn to another important result. 


\section{UNIQUENESS OF PLANCK'S CONSTANT}

In the standard definition [23] of TP of two (associative) algebras $\left\{A_{k}, \mu_{k}, \mathbb{F}\right\},(k=1,2)$ where $\mu_{k}$ is the product law and $\mathbb{F}$ is the field, one has the composition law

$$
\mu_{12}=\left(\mu_{1} \otimes \mu_{2}\right) \circ S
$$

defined in the TP space $A_{1} \otimes A_{2}$. Let $e_{k}$ be the unit element of $A_{k}$, i.e., $(f \mu e)_{k}=(e \mu f)_{k}=f_{k}$. Restriction of the product $\mu_{12}$ to the component $A_{1}$, denoted $\mu_{12} \mid A_{1}$ should result in the product $\mu_{1} \otimes e_{2}$. This means, for two elements $f_{1} \otimes e_{2}, g_{1} \otimes e_{2} \in A_{1} \otimes e_{2}$, we have

$$
\begin{aligned}
\left(f_{1} \otimes e_{2}\right) \mu_{12}\left(g_{1} \otimes e_{2}\right) & =(f \mu g)_{1} \otimes(e \mu e)_{2} \\
& =(f \mu g)_{1} \otimes e_{2} .
\end{aligned}
$$

Similarly, $\mu_{12} \mid A_{2}=e_{1} \otimes \mu_{2}$.

Extending the above restriction requirement to the HA, we note that $\alpha$ being a Lie product, does not have an unit element and the Lie product of any element with $e$ (the unit element of the $\sigma$ product) vanishes, i.e., $f \alpha e=0$ Algebraically, this means that the HA is central, i.e., if $f \alpha x=0$ holds for arbitrary $f \in \mathcal{H}$, then $x=c \sigma e$ where $c \in \mathbb{R}$. We are now in a position to prove

Theorem 2 There can be only one PC.

Proof: Consider the TP $\mathcal{A}^{a_{12}}$ of the associative enveloping algebras of two QHA's $\mathcal{A}^{a_{1}}$ and $\mathcal{A}^{a_{2}}$. Then we have

$$
\begin{aligned}
& \tau_{12} \mid A_{1}=\tau_{1} \otimes e_{2}, \\
& \tau_{12} \mid A_{2}=e_{1} \otimes \tau_{2} .
\end{aligned}
$$

Using the relations (192324) and equating the symmetric and antisymmetric parts separately, we obtain

$$
\begin{aligned}
\alpha_{12} \mid A_{1} & =\sqrt{\frac{a_{1}}{a_{12}}}\left(\alpha_{1} \otimes e_{2}\right), \\
\alpha_{12} \mid A_{2} & =\sqrt{\frac{a_{2}}{a_{12}}}\left(e_{1} \otimes \alpha_{2}\right) .
\end{aligned}
$$


wherein we have used the relation $(e \sigma e)_{k}=e_{k}$. We now invoke the standard restriction requirements:

$$
\begin{aligned}
& \alpha_{12} \mid A_{1}=\left(\alpha_{1} \otimes e_{2}\right), \\
& \alpha_{12} \mid A_{2}=\left(e_{1} \otimes \alpha_{2}\right) .
\end{aligned}
$$

Comparison of Eqs.(42) and (43) leads to the result

$$
a_{12}=a_{1}=a_{2}=a \quad \text { (say) }
$$

and hence,

$$
\hbar_{12}=\hbar_{1}=\hbar_{2}=\hbar \text {. }
$$

The result that $\mathrm{PC}$ is unique, apart from reaffirming the conventional assumption, also referee 1 demonstrates clearly the very consistency of the HA approach.

\section{CONCLUSION}

To summarize, we have followed an algebraic approach to QM which is free of the positionmomentum generators satisfying the Heisenberg commutation relation. It gives importance to the CR (13) relating both the Lie and the Jordan products. In this way the concept of a QHA subsumes symplectic (i.e., position-momentum generated algebra) as well as nonsymplectic (i.e., algebras for internal degrees of freedom such as spin) variables. We first show that two QHA's with different PC's can lead to a composite QHA with yet another PC. We then show that a QHA can form a composite with a CHA resulting in a QHA and thus

allowing for the only consistent description of a mixed mechanics. However, back-reaction of the quantum system on the classical is shown to be ruled out in this scheme. This result has important bearings on quantum measurement issues as there is no way to describe a quantum system and a (classical) measuring apparatus in a consistent way in the sense of Bohr. We have also proved, based on the natural restriction requirement, that there is but one $\mathrm{PC}$.

f In the light of our first result regarding freezing of dynamics in the classical-quantum system interaction, one may wonder how the model such as the one proposed recently by Belavkin[16] is able to deal with the measurement problem. The answer to this puzzle lies 
in the simple fact that in that model (as in the original von Neumann[17]) the apparatus is also assumed to have a wave function (or a wave packet) thus endowing it with quantum property. The model is based on Schrödinger picture and also one considers only the Lie evolution (dynamics) mediated by stochastic interaction and dissipation. The Jordan product ( $\sigma^{a}$ in our notation) of observables does not enter at all in the treatment of ref. [16]. For example, given two observables $f$ and $g$, their "observable" product $f \sigma^{a} g$ evolves (say, under a Hamiltonian $h)$ according to the product $h \alpha^{a}\left(f \sigma^{a} g\right)$. So also the observables $f$ and $g$ evolve under the same $h$. Consistency of time evolution requires the derivation law (12) to hold. Requirements such as this need to be satisfied by the observables of the coupled systems also. It is not possible to make such consistency checks in the Schrödinger picture adopted in ref. [16].

1 The concept of HA as introduced here pertains to Bose systems. There is a Fermionic counterpart. It has been introduced in ref[2]. In this case one needs a graded algebra structure and the identities defining the algebra are graded versions of the identities (9, 13). Results derived in the present work can be extended to Fermi HA's. This extension along with some results concerning composition of Bose and Fermi Hamilton algebras will be dealt with in a separate work.

\section{Acknowledgments}

The author wishes to thank the Director, Institute of Physics, Bhubaneswar, for his kind hospitality. He would also like to express his thanks to Dr Arun Kumar Pati for his comments on the manuscript. 


\section{APPENDIX A: PROOF OF LEMMA 4}

We compute first the lhs of the identity:

$$
\begin{aligned}
& (f \alpha(g \sigma h))_{12}=f_{12} \alpha_{12}\left\{(g \sigma h)_{1}(g \sigma h)_{2}-\sqrt{a_{1} a_{2}}(g \alpha h)_{1}(g \alpha h)_{2}\right\} \\
& =\sqrt{\frac{a_{1}}{a_{12}}}(f \alpha(g \sigma h))_{1}(f \sigma(g \sigma h))_{2}+\sqrt{\frac{a_{2}}{a_{12}}}(f \sigma(g \sigma h))_{1}(f \alpha(g \sigma h))_{2} \\
& -a_{1} \sqrt{\frac{a_{2}}{a_{12}}}(f \alpha(g \alpha h))_{1}(f \sigma(g \alpha h))_{2}+a_{2} \sqrt{\frac{a_{1}}{a_{12}}}(f \sigma(g \alpha h))_{1}(f \alpha(g \alpha h))_{2} \\
& =\sqrt{\frac{a_{1}}{a_{12}}}((f \alpha g) \sigma h)_{1}(f \sigma(g \sigma h))_{2}+\sqrt{\frac{a_{1}}{a_{12}}}(g \sigma(f \alpha h))_{1}(f \sigma(g \sigma h))_{2} \\
& +\sqrt{\frac{a_{2}}{a_{12}}}(f \sigma(g \sigma h))_{1}((f \alpha g) \sigma h)_{2}+\sqrt{\frac{a_{2}}{a_{12}}}(f \sigma(g \sigma h))_{1}(g \sigma(f \alpha h))_{2} \\
& -a_{1} \sqrt{\frac{a_{2}}{a_{12}}}((f \alpha g) \alpha h)_{1}(f \sigma(g \alpha h))_{2}+a_{1} \sqrt{\frac{a_{2}}{a_{12}}}(g \alpha(f \alpha h))_{1}(f \sigma(g \alpha h))_{2} \\
& -a_{2} \sqrt{\frac{a_{1}}{a_{12}}}(f \sigma(g \alpha h))_{1}((f \alpha g) \alpha h)_{2}+a_{2} \sqrt{\frac{a_{1}}{a_{12}}}(f \sigma(g \alpha h))_{1}(g \alpha(f \alpha h))_{2}
\end{aligned}
$$

The two terms on the rhs of the identity are:

$$
\begin{aligned}
& (g \sigma(f \alpha h))_{12}=g_{12} \sigma_{12}\left[\sqrt{\frac{a_{1}}{a_{12}}}(f \alpha h)_{1}(f \sigma h)_{2}+\sqrt{\frac{a_{2}}{a_{12}}}(f \sigma h)_{1}(f \alpha h)_{2}\right] \\
& =\sqrt{\frac{a_{1}}{a_{12}}}(g \sigma(f \alpha h))_{1}(g \sigma(f \sigma h))_{2}+\sqrt{\frac{a_{2}}{a_{12}}}(g \sigma(f \sigma h))_{1}(g \sigma(f \alpha h))_{2} \\
& -a_{1} \sqrt{\frac{a_{2}}{a_{12}}}(g \alpha(f \alpha h))_{1}\left[((g \alpha f) \sigma h)_{2}+(f \sigma(g \alpha h))_{2}\right] \\
& -a_{2} \sqrt{\frac{a_{1}}{a_{12}}}\left[((g \alpha f) \sigma h)_{1}+(f \sigma(g \alpha h))_{1}\right](g \alpha(f \alpha h))_{2}
\end{aligned}
$$

and

$$
\begin{aligned}
& ((f \alpha g) \sigma h)_{12}=\left[\sqrt{\frac{a_{1}}{a_{12}}}(f \alpha g)_{1}(f \sigma g)_{2}+\sqrt{\frac{a_{2}}{a_{12}}}(f \sigma g)_{1}(f \alpha g)_{2}\right] \sigma_{12} h_{12} \\
& =\sqrt{\frac{a_{1}}{a_{12}}}((f \alpha g) \sigma h)_{1}((f \sigma g) \sigma h)_{2}+\sqrt{\frac{a_{2}}{a_{12}}}((f \sigma g) \sigma h)_{1}((f \alpha g) \sigma h)_{2} \\
& -a_{1} \sqrt{\frac{a_{2}}{a_{12}}}((f \alpha g) \alpha h)_{1}\left[((f \alpha h) \sigma g)_{2}+(f \sigma(g \alpha h))_{2}\right] \\
& -a_{2} \sqrt{\frac{a_{1}}{a_{12}}}\left[((f \alpha h) \sigma g)_{1}+(f \sigma(g \alpha h))_{1}\right]((f \alpha g) \alpha h)_{2} .
\end{aligned}
$$


We now simplify the combination

$$
\begin{aligned}
& \left.(f \alpha(g \sigma h))_{12}-(g \sigma(f \alpha h))_{12}-(f \alpha g) \sigma h\right)_{12} \\
& =-\sqrt{\frac{a_{1}}{a_{12}}}((f \alpha g) \sigma h)_{1}\left(\Delta_{\sigma}(f, g, h)\right)_{2}-\sqrt{\frac{a_{2}}{a_{12}}}\left(\Delta_{\sigma}(f, g, h)\right)_{1}((f \alpha g) \sigma h)_{2} \\
& +\sqrt{\frac{a_{1}}{a_{12}}}(g \sigma(f \alpha h))_{1}\left(\Delta_{\sigma}(g, h, f)\right)_{2}+\sqrt{\frac{a_{2}}{a_{12}}}\left(\Delta_{\sigma}(g, h, f)\right)_{1}(g \sigma(f \alpha h))_{2} \\
& +a_{1} \sqrt{\frac{a_{2}}{a_{12}}}((f \alpha g) \alpha h)_{1}((f \alpha h) \sigma g)_{2}+a_{2} \sqrt{\frac{a_{1}}{a_{12}}}((f \alpha h) \sigma g)_{1}((f \alpha g) \alpha h)_{2} \\
& +a_{1} \sqrt{\frac{a_{2}}{a_{12}}}(g \alpha(f \alpha h))_{1}((g \alpha f) \sigma h)_{2}+a_{2} \sqrt{\frac{a_{1}}{a_{12}}}((g \alpha f) \sigma h)_{1}(g \alpha(f \alpha h))_{2} .
\end{aligned}
$$

Making use of the CR (13) in the associators occurring in the above relation, we immediately see that the rhs vanishes thereby establishing the derivation identity. 


\section{APPENDIX B: PROOF OF LEMMA 5}

We first simplify the individual terms of the relation.

$$
\begin{aligned}
( & (f \sigma g) \sigma h)_{12} \\
= & {\left[(f \sigma g)_{1}(f \sigma g)_{2}-\sqrt{a_{1} a_{2}}(f \alpha g)_{1}(f \alpha g)_{2}\right] \sigma h_{12} } \\
= & ((f \sigma g) \sigma h)_{1}((f \sigma g) \sigma h)_{2}-\sqrt{a_{1} a_{2}}((f \sigma g) \alpha h)_{1}((f \sigma g) \alpha h)_{2} \\
& -\sqrt{a_{1} a_{2}}((f \alpha g) \sigma h)_{1}((f \alpha g) \sigma h)_{2}+a_{1} a_{2}((f \alpha g) \alpha h)_{1}((f \alpha g) \alpha h)_{2} \\
= & ((f \sigma g) \sigma h)_{1}((f \sigma g) \sigma h)_{2} \\
& -\sqrt{a_{1} a_{2}}\left\{((f \alpha h) \sigma g)_{1}+(f \sigma(g \alpha h))_{1}\right\}\left\{((f \alpha h) \sigma g)_{1}+(f \sigma(g \alpha h))_{2}\right\} \\
& -\sqrt{a_{1} a_{2}}((f \alpha g) \sigma h)_{1}((f \alpha g) \sigma h)_{2}+\left(\Delta_{\sigma}(g, h, f)\right)_{1}\left(\Delta_{\sigma}(g, h, f)\right)_{2} \\
= & ((f \sigma g) \sigma h)_{1}((f \sigma g) \sigma h)_{2}-\sqrt{a_{1} a_{2}}((f \alpha h) \sigma g)_{1}\left((f \alpha h)_{\sigma g}\right)_{2} \\
& -\sqrt{a_{1} a_{2}}((f \alpha h) \sigma g)_{1}(f \sigma(g \alpha h))_{2}-\sqrt{a_{1} a_{2}}(f \sigma(g \alpha h))_{1}((f \alpha h) \sigma g)_{2} \\
& -\sqrt{a_{1} a_{2}}(f \sigma(g \alpha h))_{1}(f \sigma(g \alpha h))_{2}-\sqrt{a_{1} a_{2}}((f \alpha g) \sigma h)_{1}((f \alpha g) \sigma h)_{2} \\
& +\left(\Delta_{\sigma}(g, h, f)\right)_{1}(\Delta \sigma(g, h, f))_{2} \\
= & ((f \sigma g) \sigma h)_{1}((f \sigma g) \sigma h)_{2}+((g \sigma h) \sigma f)_{1}((g \sigma h) \sigma f)_{2} \\
& -((g \sigma h) \sigma f)_{1}(g \sigma(h \sigma f))_{2}-(g \sigma(h \sigma f))_{1}((g \sigma h) \sigma f)_{2} \\
& +(g \sigma(h \sigma f))_{1}(g \sigma(h \sigma f))_{2} \\
& -\sqrt{a_{1} a_{2}}((f \alpha h) \sigma g)_{1}((f \alpha h) \sigma g)_{2}-\sqrt{a_{1} a_{2}}((f \alpha h) \sigma g)_{1}(f \sigma(g \alpha h))_{2} \\
& -\sqrt{a_{1} a_{2}}(f \sigma(g \alpha h))_{1}((f \alpha h) \sigma g)_{2}-\sqrt{a_{1} a_{2}}(f \sigma(g \alpha h))_{1}(f \sigma(g \alpha h))_{2} \\
& -\sqrt{a_{1} a_{2}}((f \alpha g) \sigma h)_{1}((f \alpha g) \sigma h)_{2} . \\
& (f \sigma g) \\
&
\end{aligned}
$$

Here in the second equality, the derivation relation (12) and the CR (13) have been used and in the fourth equality the associator expressions have been explicitly substituted using 
(13). Similar simplification steps lead to the result

$$
\begin{aligned}
& (f \sigma(g \sigma h))_{12} \\
& =(f \sigma(g \sigma h))_{1}(f \sigma(g \sigma h))_{2}+((h \sigma f) \sigma g)_{1}((h \sigma f) \sigma g)_{2} \\
& \quad-((h \sigma f) \sigma g)_{1}(h \sigma(f \sigma g))_{2}-(h \sigma(f \sigma g))_{1}((h \sigma f) \sigma g)_{2} \\
& \quad+(h \sigma(f \sigma g))_{1}(h \sigma(f \sigma g))_{2} \\
& \quad-\sqrt{a_{1} a_{2}}((f \alpha g) \sigma h)_{1}((f \alpha g) \sigma h)_{2}-\sqrt{a_{1} a_{2}}((f \alpha g) \sigma h)_{1}(g \sigma(f \alpha h))_{2} \\
& \quad-\sqrt{a_{1} a_{2}}(g \sigma(f \alpha h))_{1}((f \alpha g) \sigma h)_{2}-\sqrt{a_{1} a_{2}}(g \sigma(f \alpha h))_{1}(g \sigma(f \alpha h))_{2} \\
& \quad-\sqrt{a_{1} a_{2}}(f \sigma(g \alpha h))_{1}(f \sigma(g \alpha h))_{2} .
\end{aligned}
$$

The remaining term of the $\mathrm{CR}$ is

$$
\begin{aligned}
a_{12} & ((f \alpha h) \alpha g)_{12} \\
= & a_{12}\left[\sqrt{\frac{a_{1}}{a_{12}}}(f \alpha h)_{1}(f \sigma h)_{2}+\sqrt{\frac{a_{1}}{a_{12}}}(f \alpha h)_{1}(f \sigma h)_{2}\right] \alpha_{12}\left(g_{12}\right) \\
= & a_{12} \sqrt{\frac{a_{1}}{a_{12}}}\left[\sqrt{\frac{a_{1}}{a_{12}}}((f \alpha h) \alpha g)_{1}((f \sigma h) \sigma g)_{2}+\sqrt{\frac{a_{2}}{a_{12}}}((f \alpha h) \sigma g)_{1}((f \sigma h) \alpha g)_{2}\right] \\
& +a_{12} \sqrt{\frac{a_{2}}{a_{12}}}\left[\sqrt{\frac{a_{1}}{a_{12}}}((f \sigma h) \alpha g)_{1}((f \alpha h) \sigma g)_{2}+\sqrt{\frac{a_{2}}{a_{12}}}((f \sigma h) \sigma g)_{1}((f \alpha h) \alpha g)_{2}\right] \\
= & a_{1}((f \alpha h) \alpha g)_{1}((f \sigma h) \sigma g)_{2}+\sqrt{a_{1} a_{2}}((f \alpha h) \sigma g)_{1}\left[((f \alpha g) \sigma h)_{2}+(f \sigma(h \alpha g))_{2}\right] \\
& +\sqrt{a_{1} a_{2}}\left[((f \alpha g) \sigma h)_{1}+(f \sigma(h \alpha g))_{1}\right]((f \alpha h) \sigma g)_{2} \\
& +a_{2}((f \sigma h) \sigma g)_{1}((f \alpha h) \alpha g)_{2} \\
= & \left(\Delta_{\sigma}(f, g, h)\right)_{1}((f \sigma h) \sigma g)_{2}+\sqrt{a_{1} a_{2}}((f \alpha h) \sigma g)_{1}((f \alpha g) \sigma h)_{2} \\
& +\sqrt{a_{1} a_{2}}((f \alpha h) \sigma g)_{1}(f \sigma(h \alpha g))_{2}+\sqrt{a_{1} a_{2}}((f \alpha g) \alpha h)_{1}((f \alpha h) \sigma g)_{2} \\
& +\sqrt{a_{1} a_{2}}(f \sigma(h \alpha g))_{1}((f \alpha h) \sigma g)_{2}+((f \sigma h) \sigma g)_{1}\left(\Delta_{\sigma}(f, g, h)\right)_{2} \\
= & ((f \sigma g) \sigma h)_{1}((f \sigma h) \sigma g)_{2}-(f \sigma(g \sigma h))_{1}((f \sigma h) \sigma g)_{2} \\
& +((f \sigma h) \sigma g)_{1}((f \sigma g) \sigma h)_{2}-((f \sigma h) \sigma g)_{1}(f \sigma(g \sigma h))_{2} \\
& +\sqrt{a_{1} a_{2}}((f \alpha h) \sigma g)_{1}((f \alpha g) \sigma h)_{2}+\sqrt{a_{1} a_{2}}((f \alpha h) \sigma g)_{1}(f \sigma(h \alpha g))_{2} \\
& +\sqrt{a_{1} a_{2}}((f \alpha g) \sigma h)_{1}((f \alpha h) \sigma g)_{2}+\sqrt{a_{1} a_{2}}(f \sigma(h \alpha g))_{1}((f \alpha h) \sigma g)_{2} .
\end{aligned}
$$

Combining these three terms leads to the final result

$$
((f \sigma g) \sigma h)_{12}-(f \sigma(g \sigma h))_{12}-a_{12}((f \alpha h) \alpha g)_{12}=0
$$


i.e., $\quad \Delta_{\sigma}(f, g, h)_{12}=a_{12}((f \alpha h) \alpha g)_{12}$.

[1] Grgin E and Petersen A (1974) J. Math. Phys. 15764.

[2] Sahoo D (1977) Pramana 8545.

[3] Bohr N (1927) Atti del Congresso Internazionale dei Fisici, Como,; Reprinted: (1928) Nature (London) 121 78, 580; Bohr N (1939) New Theories in Physics (International Institute of Intellectual Cooperation, Paris) pp. 11-45.

[4] Sahoo D (1972) Some Algebraic Structure of the Relationship between Classical and Quantum Mechanics, Ph.D. Thesis, Belfer Graduate School of Science, Yeshiva University, New York.

[5] Anderson A (1995) Phys. Rev. Lett. 74621.

[6] Boucher W and Traschen J (1988) Phys. Rev. D 373522.

[7] Salcedo L L (1996) Phys. Rev. A 543657.

[8] Caro J and Salcedo L L (1999) Phys. Rev., A 60842.

[9] Aleksandrov I V (1981) Z. Naturforsch., A 36902.

[10] Prezhdo O V and Kisil V (1997) Phys. Rev., A 56162.

[11] Dirac P A M (1973) Quantum Mechanics, Oxford University Press, London.

[12] Anderson A (1995) LANL e-print quant-ph/9511014.

[13] Diosi L, Gisin N and Strunz W T (2000) Phys Rev A 61 022108; Diosi L and Halliwell J J LANL e-print quant-ph/9705008; Diosi L LANL e-prints quant-ph/9510028; quant-ph/9503023, quant-ph/9903088 quant-ph/990208.

[14] Hay O and Peres A (1998) Phys. Rev., A 58116.

[15] Peres A and Temo R (2001) Phys. Rev., A 63022101.

[16] Belavkin V P and Melsheimer(1996) Semiclass. Opt., 8 167; Belavkin V P and Stratonovich R L (1996) Inter. Jour. Theor. Phys., 35 2215; Belavkin V P (1989) Phys. Lett., A 140 3; Belavkin V P and Staszewski P (1989) Phys Lett, A 140 359; Belavkin V P (1990) J. Math. Phys., 31 2930; Belavkin V P (2002) Rep. Prog. Phys., 65353.

[17] Von Neumann J (1955), Mathematical Foundations in quantum Mechanics, Princeton University Press, New Jersey.

[18] Sudarshan E C G (1976) Pramana 6 117; Sherry T N and Sudarshan E C G (1978) Phys. Rev. D 18 4580, Phys. Rev. D 20 857; Gautam S R, Sherry T N and Sudarshan E C G (1979) 
Phys. Rev. D 203081.

[19] Wichmann E H (1971) Berkeley Physics Course, McGraw-Hill, New York, Vol. 4 pp. 196-200.

[20] Fischback E, Greene G L and Hughes R J (1991) Phys. Rev. Lett. 66256.

[21] Battaglia F (1993) Inter. Jour. Theor. Phys., 321401.

[22] Emch G G (1973) Algebraic Methods in Statistical Mechanics and Quantum Field Theory, Wiley-Interscience, New York.

[23] Greub W (1976)Multilinear Algebra, Springer-Verlag, New York.

[24] Schafer R D (1966) An Introduction to Nonassociative Algebras, Academic Press, New York. 